\title{
Apnea Training Specificity and its Implications for Performance in Aquatic Sports: Case Study Reports
}

\section{Sylvia Konstantinidou* and Chrysoula Chairopoulou}

\section{School of Physical Education \& Sports Sciences, National \& Kapodistrian University of Athens, Greece}

*Corresponding author: Sylvia Konstantinidou, School of Physical Education and Sports Sciences, National \& Kapodistrian University of Athens, Division of Aquatic Sports, Ethnikis Antistasis 41, Dafni 17237, Athens, Greece, E-mail: sylvia_konst@phed.uoa.gr

\begin{abstract}
Apnea training forms an essential drill used by athletes in aquatic sports such as diving, underwater rugby but also in swimming particularly fin and synchronized swimming. Literature suggests a number of long-term adaptations observed in apnea-conditioned athletes compared to controls. Unique responses to acute apneas have also been manifested by athletes with minimum apnea exposure though arguably to a lesser extent. The actual benefits of apnea training on athlete's performance in aquatic sports have not yet been fully established. Perhaps, the type of apnea training might determine its impact on performance contingent to sport particularities. Three pilot case studies concerning Sport Science student responses to alternative forms of apnea after following a $13 \pm 2$ day apnea-training protocol is presented. Results suggest that the specificity of apnea drills might provide an explanation of the inconclusive results on apnea training benefits in aquatic sports.
\end{abstract}

\section{Keywords}

Apnea training, Aquatic sports performance, Hypoxia

\section{Introduction}

Apnea training constitutes a form of hypoxic training; the latter being applied to a wide diversity of sports demanding aerobic capacity though results concerning its importance are inconclusive [1]. Hypoxia relates to reduced Oxygen $\left(\mathrm{O}_{2}\right)$ in the environmental air due to the fall of its partial pressure i.e. $\mathrm{PO}_{2}<150 \mathrm{mmHg}$ [2] caused by a fall of the barometric Pressure (Pb)-Boyle's Law. For example an ascent at high altitude $(\mathrm{Pb}<760$ $\mathrm{mmHg}$ ) exposes us to hypoxia leading to hypoxemia i.e. reduced $\mathrm{O}_{2}$ circulating in our blood [3]. However, divers breathing air with $\mathrm{O}_{2}$ concentration $12.67 \%$ are also experiencing hypoxia corresponding to an elevation level of $\sim 4,500 \mathrm{~m}(460 \times 20.93 \% / 760=12.67 \%)$.

Apnea is allegedly trainable as manifested in extended apnea duration supplemented by stronger diving response-an oxygen conservation mechanism due to conditioning. In the long-term, apnea training may enhance individuals' aerobic capacity through improved aerobic enzymes, increased capillary vessels and mitochondria [4]. However, both the trainability of apnea and its benefits with respect to performance on aquatic sports are not clearly established particularly for competitive events at sea level [5].

Three Sports Science students at the University of Athens, one male, two females, accustomed to apnea to a different degree, volunteered to participate in a pilot study aimed at testing the trainability of apnea and its potential implications on sports performance. Considering the long duration of synchronized swimmers' training sessions, we wanted to test whether a quick dry apnea drill to be applied at their spare time, could be potentially beneficial for their performance. Study participants followed a $13 \pm 2$ day of Dry Apnea Training Programme (DATP) exhibiting diverse apnea duration improvements as measured under alternative testing conditions. However, all participants manifested relatively higher apnea duration increases under «dry apnea in supine position». Results support the apnea-training specificity argument as participants performed maximum breath hold sessions lying in a supine position. 
Further research is warranted using alternative apnea training protocols but also examining the potential longterm physiological impact of apnea-conditioning on individuals' aerobic capacity and anaerobic power.

\section{Methods}

Study participants were briefed and asked to complete a health screening questionnaire before giving their written consent to participate in the study [6]. The study was conducted in accordance with the Declaration of Helsinki and following School's ethical approval. We measured and recorded individual's anthropometric variables (SECA, USA) and assessed their fitness level [7] and anxiety status in general and before apnea sessions. Anxiety assessments were performed using two scales validated in the Greek population namely, the STAI-Trait and the STAI-State (Form X) [8-10].

Study participants were asked to follow a supervised daily Dry Apnea Training Protocol (DATP) consisting of five consecutive maximum apneas lying in a supine position with a two-minute rest interval in between apnea bouts, for a period of two weeks [4]. Despite the minimum duration of the DATP considering its analogy to altitude acclimatization it is acceptable for the purposes of a preliminary investigation [11]. Apnea durations in alternative testing conditions (static/dynamic and dry/wet) were measured before (baseline) and after the DATP which lasted for $13 \pm 2$ days. After each apnea trial, a 3-minute rest interval (Speedo stopwatch, SD50588, China) elapsed during which participants indicated their level of Perceived Exertion (RPE) and their Perceived Dyspnea (RPD - modified Borg's scale [12]. Arterial blood pressure (Fora P91, For a Care Suisse AG), oxygen saturation (pulse oximeter A320-OPE, Ver. 5.1, Inter fox, Greece) and heart rates (Polar, S610i, Finland) were recorded within the last 10 seconds before and after each apnea. All apnea trials were conducted at the School's indoor $25 \mathrm{~m}$ swimming pool with water temperature $26.2 \pm 0.2{ }^{\circ} \mathrm{C}$ and ambient temperature at 25 $\pm 1{ }^{\circ} \mathrm{C}$. Anthropometric measurements were conducted at the Schools' Aquatics Division Laboratory while fitness tests were performed in the Apparatus Gymnastics room. Running tests were conducted at the Schools outdoors track and field.

\section{Potential benefits of Hypoxic training for Aquat- ic Sports}

Voluntary apnea bouts, particularly involving face immersion may trigger the diving reflex characterized by bradycardia, a fall in the cardiac output and peripheral blood flow leading to oxygen conservation for vital organs [13]. The diving reflex, as a survival mechanism, is accompanied with an elevated arterial blood pressure though results are inconclusive $[14,15]$. The lack of oxygen due to apnea, inevitably triggers the anaerobic metabolism causing an increase in blood lactate $[13,16$ 18]. However, apnea trained individuals exhibit reduced blood acidosis and oxidative stress which may positively affect performance $[17,19]$. Further, apnea triggers a temporary rise in haemoglobin which is higher in divers compared to skiers and controls [20]. Actually, apnea triggers spleen contraction and a consequent rise in EPO and red blood cells [21,22]. This "artificial" elevation of hemoglobin and hematocrit lasts approximately for ten minutes which suffice for boosting swimmers' performance when applied prior to a race [23].

Apnea training apparently prompts higher apnea duration [20] but also, higher tolerance to hypoxic conditions [24]. This may be attributed to athletes' increased mitochondria, capillary vessels and aerobic enzymes, leading to an increased $\mathrm{VO}_{2 \max }$ and $\mathrm{O}_{2}$ saturation $[25,26]$. Overall, elite divers develop protective mechanisms against hypoxia including a reduced oxygen consumption compared to controls.

Results on dynamic apnea training on swimmers [27] and hypoxic training [28] have shown no discernible effect on performance. However, improved swimming technique and $\mathrm{VO}_{2 \max }$ have been recorded respectively. In contrast, others report improved performance for swimmers that undertook hypoxic training $[29,30]$. Further, evidence suggests that apnea training improves respiratory muscle performance by delaying fatigue during continuous exercise at maximum effort [31]. Apparently, apnea training causes physiological adaptations allowing individuals not only to exert an improved breath control but also, to cope with conditions of hypoxia in an effective and efficient manner although contradictory evidence exists [32-34].

This study purports to determine whether apnea is trainable using an apnea drill, with a view to establishing its potential benefits for improved performance in aquatic sports and particularly in synchronized swimming where repeated bouts of short dynamic apneas are prevalent during competitive events.

\section{Case Series}

\section{Case 1}

AP a 29-year-old male is a folklore dancing teacher and a recreational diver both in free and autonomous diving for the past 3 years. Upon completion of the DATP for 2 weeks his dry apnea duration more than tripled. Although his dynamic apnea duration showed minimal improvement compared to static apneas, his apnea swimming distance increased considerably (Table 1). Table 2 presents AP's noticeable heart rate drop and arterial blood pressure rise after apnea periods compared to rest i.e. before apneas. However, his heart rate at baseline was relatively elevated possibly due to stress. In fact, his anxiety level before apnea trials was above average with a STAI-State score 51 although his STAI-Trait i.e. stress condition in general (completed on a different day) was 42 (scales ranging from 20 to 80 ). Further, although AP indicated a relatively high level of 
Table 1: Apnea durations of AP before and after DATP performed with a 3-minute rest interval between each apnea type. During static apneas (DA_SUP \& WA_FI) a nose clip was used.

\begin{tabular}{|l|l|l|l|l|l|l|}
\hline & DA_SUP & WA_FI & DYA_FS & \multicolumn{3}{|l|}{ DYA_US } \\
\cline { 2 - 4 } & $\mathbf{( s e c )}$ & $\mathbf{s e c})$ & Sec & Meters & Sec & Meters \\
\hline Before DATP & 40 & 57 & 45 & 45 & 26 & 25 \\
\hline After DATP & 149 & 156 & 45 & 50 & 34 & 37.5 \\
\hline Difference & $109(273 \%)$ & $99(174 \%)$ & 0 & $5(11 \%)$ & $8(31 \%)$ & $12.5(50 \%)$ \\
\hline
\end{tabular}

DA_SUP: Dry Apnea in a Supine Position; WA_FI: Wet Apnea with Face Immersion; DYA_FS: Dynamic Apnea Freestyle Swimming on Water Surface; DYA_US: Dynamic Apnea Underwater Swimming.

Table 2: Cardiovascular variables and perceived exertion/dyspnea measurements of AP after DATP.

\begin{tabular}{|c|c|c|c|c|c|c|c|c|c|c|c|c|}
\hline & \multicolumn{3}{|c|}{ DA_SUP } & \multicolumn{3}{|c|}{ WA_FI } & \multicolumn{3}{|c|}{ DYA_US } & \multicolumn{3}{|c|}{ DYA_FS } \\
\hline & BFA & AFA & DF (\%) & BFA & AFA & DF (\%) & BFA & AFA & DF (\%) & BFA & AFA & DF (\%) \\
\hline SBP (mmHg) & 110 & 120 & 9 & 110 & 115 & 4.5 & 110 & 130 & 18 & 110 & 125 & 13.6 \\
\hline DBP (mmHg) & 90 & 90 & 0 & 80 & 80 & 0 & 90 & 90 & 0 & 90 & 90 & 0 \\
\hline HR (bits- $\min ^{-1}$ ) & 88 & 80 & -9 & 90 & 58 & -36 & 97 & 59 & -39 & 109 & 91 & -16.5 \\
\hline RPD (0-10) & \multicolumn{3}{|l|}{0} & \multicolumn{3}{|l|}{0} & \multicolumn{3}{|l|}{0} & \multicolumn{3}{|l|}{0} \\
\hline RPE (6-20) & \multicolumn{3}{|l|}{18} & \multicolumn{3}{|l|}{17} & \multicolumn{3}{|l|}{17} & \multicolumn{3}{|l|}{17} \\
\hline
\end{tabular}

AFA: After Apnea; BFA: Before Apnea; DF: Difference; DA_SUP: Dry Apnea in a Supine Position; WA_Fl: Wet Apnea with Face Immersion; DYA_FS: Dynamic Apnea Freestyle Swimming on Water Surface; DYA_US: Dynamic Apnea Underwater Swimming; RPD: Rate of Perceived Dyspnea; RPE: Rate of Perceived Exertion.

Table 3: Anthropometric data of AP.

\begin{tabular}{|c|c|c|c|c|c|c|c|c|c|}
\hline $\begin{array}{l}\text { Body weight } \\
\text { (Kg) }\end{array}$ & $\begin{array}{l}\text { Height } \\
(\mathrm{cm})\end{array}$ & $\begin{array}{l}\text { BMI } \\
\left(\mathrm{kg} / \mathrm{cm}^{2}\right)\end{array}$ & WHR & $\begin{array}{l}\text { Thorax inhale } \\
\text { (cm) }\end{array}$ & $\begin{array}{l}\text { Thorax exhale } \\
(\mathrm{cm})\end{array}$ & $\begin{array}{l}\text { Upper limb } \\
\text { length }(\mathrm{cm})\end{array}$ & $\begin{array}{l}\text { Lower limb } \\
\text { length }(\mathrm{cm})\end{array}$ & $\begin{array}{l}\text { Hand } \\
\text { length } \\
(\mathrm{cm})\end{array}$ & $\begin{array}{l}\text { Foot length } \\
(\mathrm{cm})\end{array}$ \\
\hline 77 & 181 & $23.5^{a}$ & $0.79^{b}$ & 98 & 91.5 & 79 & 103 & 19 & 29 \\
\hline
\end{tabular}

BMI: Body Mass Index (Kg/cm²); WHR: Waist Hip Ratio (cm/cm); a: normal [39]; b: no risk [40].

Table 4: Goodness of Fit data of AP using tests adopted from Eurofit and edited in Greek by Kleisouras [41].

\begin{tabular}{|c|c|c|c|c|c|c|}
\hline $\begin{array}{l}\text { Muscular power } \\
\text { - jump (cm) }\end{array}$ & $\begin{array}{l}\text { Muscular } \\
\text { endurance } \\
\text { (No. of abd } \\
\text { crunches) }\end{array}$ & $\begin{array}{l}\text { Flexibility } \\
\text { - waist bending } \\
\text { (cm) }\end{array}$ & $\begin{array}{l}\text { Stability } \\
\text { - stork position }\end{array}$ & $\begin{array}{l}\text { Muscular } \\
\text { endurance - } \\
\text { horizontal bar } \\
\text { (sec) }\end{array}$ & $\begin{array}{l}\text { Speed } \\
\text { - swim } 25 \\
\text { m (sec) }\end{array}$ & $\mathrm{VO}_{2 \max }-\left(\mathrm{ml} \cdot \mathrm{kg}^{-1} \cdot \mathrm{min}^{-1} .\right)^{*}$ \\
\hline 230 & 15 & 15 & 1 & 90 & 15 & 52.9 \\
\hline
\end{tabular}

${ }^{*}$ George, et al. [7].

Table 5: Apnea durations of AS before and after DATP performed with a 3-minute rest interval between each type of apnea. During static apneas (DA_SUP, WA_FI \& WA_RP) a nose clip was used.

\begin{tabular}{|l|l|l|l|l|l|}
\hline & DA_SUP (sec) & WA_FI (sec) & WA_RP (sec) & \multicolumn{2}{|l|}{ DYA_US } \\
\hline Before DATP & 75 & & & 94 & 48 \\
\hline After DATP & 132 & 86 & 110 & 50 & 40 \\
\hline Difference & $57(76 \%)$ & $60(70 \%)$ & $16(17 \%)$ & $2(4 \%)$ & $10(25 \%)$ \\
\hline
\end{tabular}

DA_SUP: Dry Apnea in Supine Position; WA_FI: Wet Apnea with Face Immersion; WA_RP: Wet Apnea in Reverse Body Position at the Pool Wall; DYA_US: Dynamic Apnea Ünderwater Swimming.

Perceived Exertion (RPE scale) he experienced no dyspnea during apnea bouts. Results are in accordance with those in the literature suggesting that the diving reflex might have been triggered.

Concerning his fitness level it was assessed at the start of the experimentation period using Cooper's test (maximum running distance at $12 \mathrm{~min}$ [35], and his classification was III/V i.e. above average. After completing the DATP he was asked to swim in the School's 25-m pool $400 \mathrm{~m}$ freestyle swimming at maximum speed. His respiratory gasses immediately after the end of the swimming session were collected using a portable spirometer (VO2000 BreezeLite, Medical Graphics Corp., U.S.A.). Using backward extrapo- lation [36], his $\mathrm{VO}_{2 \max }$ was estimated at $41.3 \mathrm{ml} \cdot \mathrm{Kg}^{-1} \cdot \mathrm{min}^{-1}$ i.e., "good" (4 out of 6, [37] which is in line with the original estimations under the running test.

Further, a number of anthropometric variables (Table 3) and fitness level variables (Table 4) were recorded before the start of the investigation as presented above [38-41].

We note normal values in terms his BMI and WHR but also concerning his goodness of fit data assessed under Eurofit test.

\section{Case 2}

AS is a 22-year-old female and a former artistic gymnast and later synchronized swimmer for over 9 years 
with participations and prizes won at national and international level. AS's estimated $\mathrm{VO}_{2 \max }$ was $36 \mathrm{ml} \cdot \mathrm{Kg}$ 1. $\mathrm{min}^{-1}$ i.e., "good" [7]. AS followed the DATP for 11 days and her apnea durations before and after the training program are listed in the Table 5.

Similar to AP, AS showed a remarkable increase in static apnea durations with the exception of the apnea in the reverse body position. It is important to note that this is a routine position that synchronized swimmers use while executing their programme during competitive events and is estimated to account for approximately $43 \%$ of total routine time [42]. Similar to AP, although her dynamic apnea duration did not show a notable improvement, her swimming distance incased noticeably. Her cardiovascular responses follow a similar pattern with AP i.e., after apnea sessions, a heart rate drop and an elevated arterial blood pressure were recorded (Table 6, Table 7 and Table 8). However, AS's baseline heart rate levels were relatively low, despite her anxiety level recorded before apnea sessions (STAI-State score 57). Her general stress condition i.e. STAI-Trait score was 48 . We also note an occasional slight drop in her oxygen saturation levels.
Additional information concerning AS is provided below.

\section{Case 3}

EK a 29-year-old female and a former swimmer with competitive experience of 13 years followed DATP for 15 days. Her estimated $\mathrm{VO}_{2 \max } 40.6 \mathrm{ml} \cdot \mathrm{kg}^{-1} \cdot \mathrm{min}^{-1}$ [7] is "very good". EK's results further confirm that the impact of apnea training is more evident in increased apnea duration under the same conditions. Her fitness level is "very good", which actually prevents us from explaining her relatively poor results after apnea training compared to the other two participants. Her reported anxiety level (STAI-State score 45) and general stress condition (STAI-Trait score 53) are also fairly normal. Furthermore, she indicated 0 on Borg's adjusted scale for dyspnea (0-10 for max dyspnea). On the other hand, she probably did not actually perform maximum apnea efforts as her average RPE scores (Borg's scale) both before and after DATP is 13.5 i.e. "somewhat difficult to difficult» (Table 9).

Being a «novice» in apnea-training relative to the other two participants, we present EK's cardiovascular

Table 6: Cardiovascular variables and perceived exertion/dyspnea measurements of AS after DATP.

\begin{tabular}{|c|c|c|c|c|c|c|c|c|c|c|c|c|}
\hline & \multicolumn{3}{|c|}{ DA_SUP } & \multicolumn{3}{|c|}{ WA_FI } & \multicolumn{3}{|c|}{ WA_RP } & \multicolumn{3}{|c|}{ DYA_US } \\
\hline & BFA & AFA & DF (\%) & BFA & AFA & DF (\%) & BFA & AFA & DF (\%) & BFA & AFA & DF (\%) \\
\hline SBP (mmHg) & 120 & 110 & -8.3 & 110 & 110 & 0 & 120 & 130 & 8.3 & 130 & 130 & 0 \\
\hline $\mathrm{DBP}(\mathrm{mmHg})$ & 80 & 90 & 12.5 & 80 & 90 & 12.5 & 80 & 90 & 12.5 & 90 & 90 & 0 \\
\hline HR (bits $\cdot \mathrm{min}^{-1}$ ) & 68 & 66 & -2.9 & 65 & 59 & -9.2 & 65 & 59 & -9.2 & 65 & 68 & 4.6 \\
\hline $\mathrm{SaO}_{2}$ & 97 & 97 & 0 & 97 & 98 & 1 & 97 & 96 & -1 & 98 & 97 & -1 \\
\hline RPD (0-10) & \multicolumn{3}{|l|}{0.5} & \multicolumn{3}{|l|}{0.5} & \multicolumn{3}{|l|}{0.5} & \multicolumn{3}{|l|}{0.5} \\
\hline RPE (6-20) & \multicolumn{3}{|l|}{15} & \multicolumn{3}{|l|}{16} & \multicolumn{3}{|l|}{11} & \multicolumn{3}{|l|}{16} \\
\hline
\end{tabular}

AFA: After Apnea; BFA: Before Apnea; DF: Difference; DA_SUP: Dry Apnea in a Supine Position; WA_FI: Wet Apnea with Face Immersion; WA_RP: Wet Apnea in Reverse Body Position; DYA_US: Dynamic Apnea Underwater Swimming; RPD: Rate of Perceived Dyspnea; RPE: Rate of Perceived Exertion.

Table 7: Anthropometric data of AS.

\begin{tabular}{|c|c|c|c|c|c|c|c|c|c|}
\hline $\begin{array}{l}\text { Body } \\
\text { weight } \\
(\mathrm{Kg})\end{array}$ & $\begin{array}{l}\text { Height } \\
(\mathrm{cm})\end{array}$ & $\begin{array}{l}\text { BMI } \\
\left(\mathrm{kg} / \mathrm{cm}^{2}\right)\end{array}$ & WHR & $\begin{array}{l}\text { Thorax } \\
\text { inhale } \\
(\mathrm{cm})\end{array}$ & $\begin{array}{l}\text { Thorax } \\
\text { exhale } \\
(\mathrm{cm})\end{array}$ & $\begin{array}{l}\text { Upper limb } \\
\text { length } \\
\text { (cm) }\end{array}$ & $\begin{array}{l}\text { Lower limb } \\
\text { length } \\
(\mathrm{cm})\end{array}$ & $\begin{array}{l}\text { Hand } \\
\text { length } \\
(\mathrm{cm})\end{array}$ & $\begin{array}{l}\text { Foot length } \\
\text { (cm) }\end{array}$ \\
\hline 61 & 161 & $23.5^{\mathrm{a}}$ & $0.68^{b}$ & 90 & 86 & 70 & 90 & 16 & 23 \\
\hline
\end{tabular}

BMI: Body Mass Index (Kg/cm²); WHR: Waist Hip Ratio (cm/cm); a: normal [39] b: no risk [40].

Table 8: Goodness of Fit data of AS using tests adopted from Eurofit and edited in Greek by Kleisouras [41].

\begin{tabular}{|c|c|c|c|c|c|c|}
\hline $\begin{array}{l}\text { Muscular power } \\
\text { - jump (cm) }\end{array}$ & $\begin{array}{l}\text { Muscular } \\
\text { endurance } \\
\text { (No. Abd } \\
\text { crunches) }\end{array}$ & $\begin{array}{l}\text { Flexibility } \\
\text { - waist } \\
\text { bending (cm) }\end{array}$ & $\begin{array}{l}\text { Stability } \\
\text { - stork position }\end{array}$ & $\begin{array}{l}\text { Muscular } \\
\text { endurance - } \\
\text { horizontal bar } \\
\text { (sec) }\end{array}$ & $\begin{array}{l}\text { Speed - swim } \\
25 \mathrm{~m}(\mathrm{sec})^{\mathrm{a}}\end{array}$ & $\begin{array}{l}\mathrm{VO}_{2 \max }-(\mathrm{ml} \cdot \mathrm{kg} \\
\left.{ }^{1} \cdot \mathrm{min}^{-1} \cdot\right)^{\mathrm{b}}\end{array}$ \\
\hline 190 & 13 & 36 & 1 & 40 & 12 & 36 \\
\hline
\end{tabular}

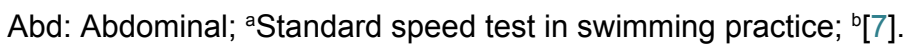

Table 9: Apnea durations of EK before and after DATP performed with a 3-minute rest interval between each type of apnea. During static apneas (DA_SUP, WA_FI \& WA_RP) a nose clip was used.

\begin{tabular}{|l|l|l|l|l|l|}
\hline & DA_SUP (sec) & WA_FI (sec) & WA_RP (sec) & \multicolumn{2}{|l|}{ DYA_US } \\
\hline Before DATP & 36 & 39 & 21 & 30 & Meters \\
\hline After DATP & 52 & 31 & 24 & 32 & 37.5 \\
\hline Difference & $16(44 \%)$ & $-8(-21 \%)$ & $3(14 \%)$ & $2(7 \%)$ & 42 \\
\hline
\end{tabular}

DA_SUP: Dry Apnea in Supine Position; WA_FI: Wet Apnea with Face Immersion; WA_RP: Wet Apnea in Reverse Body Position at the Pool Wall; DYA_US: Dynamic Apnea Ünderwater Swimming. 
variables before and after DATP expecting to find extraordinary differences. Contrary to our expectations, her heart rate before DATP slightly increased after static apneas except for the reverse body position (Table 10). There is no discernible trend on her arterial blood pressure results before DATP. These findings contradict what the diving response would have predicted i.e. brady cardia [43], and an elevated blood pressure.

EK's results after DATP (Table 11) are inconclusive concerning her heart rate while her arterial blood pressure follows the same pattern as before apnea training. As a former swimmer, EK would have some experience in apnea training which however, is not comparable to the one of AP and AS. We suspect that, her mixed results may be attributed to her lack of apnea conditioning namely, aquadicity characterized by a strong diving response accompanied by an increased lung volume and vital capacity [44]. Additional information for EK is provided below.

\section{Discussion and Conclusions}

Apnea training, similar to altitude exposure induces hypoxemia and arguably athlete's increased aerobic capacity. While athletes' stay and training at high altitude constitutes a typical tool applied in endurance-related sports, research has provided inconclusive results concerning the significance of hypoxic training. Both apnea training and altitude exposure have traditionally been used as ways of improving $\mathrm{VO}_{2 \max }$ [26]. Nonetheless, altitude exposure produces a number of risks appearing upon athletes' return at sea level including a weakened immune system, intermittent breathing during night sleep and a sustained elevated blood arterial pressure [45]. On the other hand, apnea training is potentially dangerous causing a reduced breathing drive under prolonged apnea circumstances due to athlete's conditioning in hypoxic environment [14]. Further research is required on the risks involved with apnea training as brain's safety mechanism for maintaining oxygen homeostasis is bound to collapse. Despite the po-

Table 10: Cardiovascular variables and perceived exertion/dyspnea measurements of EK before DATP.

\begin{tabular}{|c|c|c|c|c|c|c|c|c|c|c|c|c|}
\hline & \multicolumn{3}{|c|}{ DA_SUP } & \multicolumn{3}{|c|}{ WA_FI } & \multicolumn{3}{|c|}{ WA_RP } & \multicolumn{3}{|c|}{ DYA_US } \\
\hline & BFA & AFA & DF (\%) & BFA & AFA & DF (\%) & BFA & AFA & DF (\%) & BFA & AFA & DF (\%) \\
\hline SBP $(\mathrm{mmHg})$ & 100 & 110 & 10 & 100 & 100 & 0 & 110 & 110 & 0 & 110 & 125 & 13.6 \\
\hline $\mathrm{DBP}(\mathrm{mmHg})$ & 80 & 70 & -12.5 & 80 & 70 & -12.5 & 60 & 80 & 33 & 70 & 80 & 14.3 \\
\hline HR (bits- $\min ^{-1}$ ) & 56 & 67 & 19.6 & 55 & 66 & 20 & 74 & 70 & 5.4 & 75 & 61 & -18.7 \\
\hline $\mathrm{SaO}_{2}$ & 98 & 98 & 0 & 98 & 98 & 0 & 98 & 98 & 0 & 98 & 98 & 0 \\
\hline RPD $(0-10)$ & 0 & & & 0 & & & 0 & & & 0 & & \\
\hline RPE (6-20) & 9 & & & 13 & & & 15 & & & 17 & & \\
\hline
\end{tabular}

AFA: After Apnea; BFA: Before Apnea; DF: Difference; DA_SUP: Dry Apnea in a Supine Position; WA_Fl: Wet Apnea with Face Immersion; WA_RP: Wet Apnea in Reverse Body Position; DYA_US: Dynamic Apnea Underwater Swimming; RPD: Rate of Perceived Dyspnea; RPE: Rate of Perceived Exertion.

Table 11: Cardiovascular variables and perceived exertion/dyspnea measurements of EK after DATP.

\begin{tabular}{|c|c|c|c|c|c|c|c|c|c|c|c|c|}
\hline & \multicolumn{3}{|c|}{ DA_SUP } & \multicolumn{3}{|c|}{ WA_FI } & \multicolumn{3}{|c|}{ WA_RP } & \multicolumn{3}{|c|}{ DYA_US } \\
\hline & BFA & AFA & DF (\%) & BFA & AFA & DF (\%) & BFA & AFA & DF (\%) & BFA & AFA & DF (\%) \\
\hline SBP (mmHg) & 110 & 90 & 18.2 & 110 & 115 & 4.5 & 115 & 105 & -8.7 & 105 & 115 & 9.5 \\
\hline DBP $(\mathrm{mmHg})$ & 70 & 60 & -14.3 & 80 & 75 & -6.3 & 70 & 70 & 0 & 80 & 80 & 0 \\
\hline HR (bits $\cdot \mathrm{min}^{-1}$ ) & 59 & 60 & 1.7 & 65 & 64 & -1.5 & 67 & 58 & -13.4 & 59 & 61 & 3.4 \\
\hline $\mathrm{SaO}_{2}$ & 92 & 92 & 0 & 95 & 98 & 3.2 & 97 & 98 & 1 & 98 & 97 & -1 \\
\hline RPD (0-10) & 0 & & & 0 & & & 0 & & & 0 & & \\
\hline RPE (6-20) & 13 & & & 13 & & & 15 & & & 13 & & \\
\hline
\end{tabular}

AFA: After Apnea; BFA: Before Apnea; DF: Difference; DA SUP: Dry Apnea in a Supine Position; WA FI: Wet Apnea with Face Immersion; WA_RP: Wet Apnea in Reverse Body Position; DYA_US: Dynamic Apnea Underwater Swimming; RPD: Rate of Perceived Dyspnea; RPE: Rate of Perceived Exertion.

Table 12: Anthropometric data of EK.

\begin{tabular}{|c|c|c|c|c|c|c|c|c|c|}
\hline $\begin{array}{l}\text { Body weight } \\
\text { (Kg) }\end{array}$ & $\begin{array}{l}\text { Height } \\
(\mathrm{cm})\end{array}$ & $\begin{array}{l}\text { BMI } \\
\left(\mathbf{k g} / \mathbf{c m}^{2}\right)\end{array}$ & WHR & $\begin{array}{l}\text { Thorax inhale } \\
\text { (cm) }\end{array}$ & $\begin{array}{l}\text { Thorax } \\
\text { exhale (cm) }\end{array}$ & $\begin{array}{l}\text { Upper limb } \\
\text { length }(\mathbf{c m})\end{array}$ & $\begin{array}{l}\text { Lower limb } \\
\text { length }(\mathrm{cm})\end{array}$ & $\begin{array}{l}\text { Hand } \\
\text { length }(\mathrm{cm})\end{array}$ & $\begin{array}{l}\text { Foot length } \\
\text { (cm) }\end{array}$ \\
\hline 58 & 171 & $19.8^{a}$ & $0.75^{b}$ & 78 & 76 & 73 & 98 & 18 & 25 \\
\hline
\end{tabular}

BMI: Body Mass Index (Kg/cm²); WHR: Waist Hip Ratio (cm/cm); a: normal [39]; b: marginally at no risk [40].

Table 13: Goodness of Fit data of EK using tests adopted from Eurofit and edited in Greek by Kleisouras [41].

\begin{tabular}{|c|c|c|c|c|c|c|}
\hline $\begin{array}{l}\text { Muscular power } \\
\text { - jump (cm) }\end{array}$ & $\begin{array}{l}\text { Muscular } \\
\text { endurance } \\
\text { (No. Abd } \\
\text { crunches) }\end{array}$ & $\begin{array}{l}\text { Flexibility } \\
\text { - waist bending } \\
\text { (cm) }\end{array}$ & $\begin{array}{l}\text { Stability } \\
\text { - stork position }\end{array}$ & $\begin{array}{l}\text { Muscular } \\
\text { endurance - } \\
\text { horizontal bar } \\
\text { (sec) }\end{array}$ & $\begin{array}{l}\text { Speed } \\
\text { - swim } 25 \mathrm{~m} \\
(\mathrm{sec})^{\mathrm{a}}\end{array}$ & $\begin{array}{l}\mathrm{VO}_{2 \max }-(\mathrm{ml} \cdot \mathrm{kg} \\
\left.{ }^{1} \cdot \mathrm{min}^{-1} \cdot\right)^{\mathrm{b}}\end{array}$ \\
\hline 150 & 20 & 35 & $50 "$ & 28 & 13 & 40.6 \\
\hline
\end{tabular}

Abd: Abdominal; aStandard speed test in swimming practice; ${ }^{[}[7]$. 
tential dangers involved in hypoxic training, the expected benefits may outperform those risks. For example, apnea training, as a form of hypoxic training, it arguably positively affects oxidative stress and blood acidosis $[19,24]$ which may have a favorable impact on aquatic sports athletes' performance.

Results suggest that apnea can be trained and progress appears relatively fast and significant particularly for apnea-conditioned individuals, contrary to our expectations. Based on these preliminary findings, we may hypothesize that the form of apnea training determines the type of apnea on which it could have the most discernible effect. Specifically, as participants practiced dry static apneas in supine positions, their apnea duration improved mainly when executing this type of apnea. Therefore, further research is warranted examining alternative forms of apnea training including their potential impact on aerobic and anaerobic power and consequently on athletes' performance per se. Additional research may examine alternative groups accustomed to apnea to a different degree including synchronized swimmers, fin swimmers and underwater rugby players and the potential impact of sport specific apnea training protocols. For example, as synchronized swimmers are mainly using the reverse body position in the water [42] possibly, this could form their key apnea training position. Alternative apnea training protocols may involve static versus dynamic apnea either in or out of the water depending on which type of athletes are being addressed. As arguably apnea training improves aerobic endurance and anaerobic power, its benefits may also be applied to alternative sports and not necessarily only to aquatic. Consequently, comparisons could also be made on the potential impact of alternative forms of apnea training on aquatic versus non-aquatic athletes such as tri athletes, distance runners etc. and/or between alternative forms of hypoxic training including altitude exposure.

\section{References}

1. Fulco CS, Rock PB, Cymerman A (2000) Improving athletic performance: is altitude residence or altitude training helpful? Aviat Space Environ Med 71: 162-171.

2. Millet GP, Faiss R, Pialoux V (2012) Hypobaric hypoxia induces different responses than normobaric hypoxia. J Appl Physiol 3-10.

3. Semenza GL (2009) Regulation of oxygen homeostasis by hypoxia-inducible factor 1. Physiology (Bethesda) 24: 97-106.

4. Schagatay E, van Kampen M, Emanuelsson S, Holm B (2000) Effects of physical and apnea training on apneic time and the diving response in humans. Eur J Appl Physiol 82: 161-169.

5. Bärtsch P, Dehnert C, Friedmann-Bette B, Tadibi V (2008) Intermittent hypoxia at rest for improvement of athletic performance. Scand J Med Sci Sports 18: 50-56.

6. Chisholm DM, Collis MC, Kulak LL, Davenport W, Gruber $\mathrm{N}$, et al. (1975) Par-Q validation report. The evaluation of a self-administered pre-exercise screening questionnaire for adults. Unnumbered Report Prepared Jointly by BC Ministry of Health and Health and Welfare Canada.

7. George JD, Stone WJ, Burkett LN (1997) Non-exercise VO2 max estimation for physically active college students. Med Sci Sports Exerc 29: 415-423.

8. Kakkos B, Ekkekakis P, Zervas Y (1991) Psychometric analogies of the anxiety-predisposition scale of the STAI questionnaire (anxiety condition-predisposition) in the Greek population. Sports Psychol 5: 3-34.

9. Spielberger CD, Gorsuch RI, Lushere RE (1970) Manual for the State-Trait Anxiety Inventory. Palo Alto, Consultants, CA.

10. Spielberger CD, Jacobs G, Russell S, Crane RS (1983) Assessment of anger: The state-trait anger scale. Adv Personality Assessment 2: 159-187.

11. Levine BD, Stray-Gundersen J (1997) "Living high-training low": Effect of moderate-altitude acclimatization with low-altitude training on performance. J Appl Physiol (1985) 83: 102112.

12. Borg GA (1982) Psychophysical bases of perceived exertion. Med Sci Sports Exerc 14: 377-381.

13. Andersson JP, Linér MH, Fredsted A, Schagatay EK (2004) Cardiovascular and respiratory responses to apneas with and without face immersion in exercising humans. J Appl Physiol (1985) 96: 1005-1010.

14. Foster GE, Sheel AW (2005) The human diving response, its function, and its control. Scand J Med Sci Sports 15: 3-12.

15. Ferrigno M, Ferretti G, Ellis A, Warkander D, Costa M, et al. (1997) Cardiovascular changes during deep breath-hold dives in a pressure chamber. J Appl Physiol (1985) 83: 1282-1290.

16. Andersson J, Liner M, Row E, Schagatay E (2002) Diving response and arterial oxygen saturation during apnea and exercise in breath-hold divers. J Appl Physiol 93: 882-886.

17. Joulia F, Steinberg JG, Wolff F, Gavarry O, Jammes $Y$ (2002) Reduced oxidative stress and blood lactic acidosis in trained breath-hold human divers. Respir Physiol Neurobiol 133: 121-130.

18. Schagatay E, Andersson JP, Nielsen B (2007) Hematological response and diving response during apnea and apnea with face immersion. Eur J Appl Physiol 101: 125-132.

19. Joulia F, Steinberg JG, Faucher M, Jamin T, Ulmer C, et al. (2003) Breath-hold training of humans reduces oxidative stress and blood acidosis after static and dynamic apnea. Respir Physiol Neurobiol 137: 19-27.

20. Richardson M, de Bruijn R, Holmberg HC, Björklund G, Haughey $\mathrm{H}$, et al. (2005) Increase of hemoglobin concentration after maximal apneas in divers, skiers, and untrained humans. Can J Appl Physiol 30: 276-281.

21. Bakovic D, Valic Z, Eterovic D, Vukovic I, Obad A, et al. (2003) Spleen volume and blood flow response to repeated breath-hold apneas. J Appl Physiol (1985) 95: 1460-1466.

22. Espersen K, Frandsen H, Lorentzen T, Kanstrup IL, Christensen NJ (2002) The human spleen as an erythrocyte reservoir in diving-related interventions. J Appl Physiol (1985) 92: 2071-2079.

23. Lemaître F, Joulia F, Chollet D (2010) Apnea: a new training method in sport? Med Hypotheses 74: 413-415.

24. Joulia F, Lemaitre F, Fontanari $P$, Mille ML, Barthelemy $P$ (2009) Circulatory effects of apnoea in elite breath-hold divers. Acta Physiol (Oxf) 197: 75-82. 
25. Hoppeler H, Vogt M (2001) Muscle tissue adaptations to hypoxia. J Exp Biol 204: 3133-3139.

26. Nikolopoulos GA (2006) Swimming: technique, teaching, coaching. Artwork 307, Athens.

27. Lemaître F, Seifert L, Polin D, Juge J, Tourny-Chollet C, et al. (2009) Apnea training effects on swimming coordination. J Strength Cond Res 23: 1909-1914.

28. Truijens MJ, Toussaint HM, Dow J, Levine BD (2003) Effect of high-intensity hypoxic training on sea-level swimming performances. J Appl Physiol (1985) 94: 733-743.

29. Strzala M, Ostrowski A, Szygula Z (2011) Altitude training and its influence on physical endurance in swimmers. J Hum Kinet 28: 91-105.

30. Stavrou V, Toubekis AG, Karetsi E (2015) Changes in respiratory parameters and fin-swimming performance following a 16-week training period with intermittent breath holding. J Hum Kinet 49: 89-98.

31. Nygren-Bonnier M, Gullstrand L, Klefbeck B, Lindholm P (2007) Effects of glossopharyngeal pistoning for lung insufflation in elite swimmers. Med Sci Sports Exerc 39: 836-841.

32. Breskovic T, Valic Z, Lipp A, Heusser K, Ivancev V, et al. (2010) Peripheral chemoreflex regulation of sympathetic vasomotor tone in apnea divers. Clinical Autonomic Research 20: 57-63.

33. Dujic Z, Breskovic T, Bakovic D (2013) Breath-hold diving as a brain survival response. Transl Neurosci 4: 302-313.

34. Grassi B, Ferretti G, Costa M, Ferrigno M, Panzacchi A, et al. (1994) Ventilatory responses to hypercapnia and hypoxia in elite breath-hold divers. Respir Physiol 97: 323-332.
35. Cooper KH (1990) Ergometria. Symmetria Publishers.

36. Montpetit RR, Léger LA, Lavoie JM, Cazorla G (1981) VO2 peak during free swimming using the backward extrapolation of the $\mathrm{O} 2$ recovery curve. Eur J Appl Physiol Occup Physiol 47: 385-391.

37. Cooper KH (1997) The Aerobics Way. P.X. Paschalidis Medical Publishers.

38. Yamamura C, Zushi S, Takata K, Ishiko T, Matsui N, et al. (1999) Physiological characteristics of well-trained synchronized swimmers in relation to performance scores. Int $\mathrm{J}$ Sports Med 20: 246-251.

39. Wallace P (2003) Obesity as in ACSM's Exercise and chronic diseases and disabilities. In: Baltopoulos P, P.X. Paschalides, 172.

40. Williams MH (2003) Nutrition for Health, Fitness \& Sport. In: Greek Ed Syntosis, P.X. Paschalides Medical Publishers, 426435.

41. Kleisouras B (1991) Ergometria. Symmetria, 252-255.

42. Reig XI, Rodríguez-Zamora L, Clapés $P$, Barrero $A$, Chaverri D, et al. (2014) Multidimensional analysis of the structure of competitive routines in synchronized swimming. Rev Psicoldeporte 23: 173-180.

43. Alboni $P$, Alboni M, Gianfranchi L (2011) Diving bradycardia: A mechanism of defence against hypoxic damage. $J$ Cardiovasc Med (Hagerstown) 12: 422-427.

44. Varveri D, Karatzaferi C, Pollatou E, Sakkas GK (2016) Aquaticity: A discussion of the term and of how it applies to humans. J Bodyw Mov Ther 20: 219-223.

45. Bailey DM, Davies B (1997) Physiological implications of altitude training for endurance performance at sea level: a review. Br J Sports Med 31: 183-190. 